\title{
Queue Analysis for Wireless Packet Data Traffic
}

\author{
Shahram Teymori and Weihua Zhuang \\ Centre for Wireless Communications (CWC), \\ Department of Electrical and Computer Engineering, \\ University of Waterloo, Waterloo, Ontario, Canada N2L 3G1 \\ \{steymori, wzhuang\}@bbr.uwaterloo.ca
}

\begin{abstract}
Recent research based on Internet traffic measurements shows that the traffic flows have a fractal nature (i.e., self-similarity property), which causes the underestimation of the network engineering parameters when using the Poisson model. Preliminary field measurements demonstrate that packet data traffic in wireless communications also exhibits self-similarity. In this research, we investigate the queuing behavior of a self-similar traffic flow for wireless packet data applications. The traffic is modelled by an on-off source with heavy-tailed on periods. We derive a new relationship among the asymptotic distribution of the queue length, traffic specifications, wireless channel characteristics, and transmission rate. Computer simulation results are given to demonstrate the accuracy of the theoretical analysis.
\end{abstract}

Keywords: Loss probability, queueing analysis, self-similarity, wireless packet data.

\section{Introduction}

Wireless communication systems have been revolutionized by technological advances in the last decades. The third generation (and beyond) wireless networks will use the packet-switching technology to provide high-speed data services such as File Transportation Protocol (FTP) and web browsing. These applications have stringent performance requirements in terms of throughput, and transmission accuracy (packet loss rate). Data traffic flows are not sensitive to delay and can tolerate delays such as seconds and minutes, depending on the application; however, it is sensitive to packet loss probability and can normally tolerate the loss probability only up to $10^{-6}$. Selection of a proper model to analyze the queuing behaviors of network traffic flows plays an important role in performing network engineering. Recently it has been shown that the conventional Poisson traffic model is not proper for packet data traffic in the Internet [1. It is also observed that the aggregated Internet traffic flows have similar patterns in different time scales, referred to as self-similarity [2. Similarly, the existence of self-similarity in wireless network traffic flows has been observed $[3]$ and has attracted attention in the research community [4. The self-similarity 
characteristics result in that traffic engineering techniques, based on the traditional method, underestimate the required resources (such as transmission rate) to achieve quality-of-service (QoS) satisfaction.

Willinger et al. proved that the superposition of a large number of on-off traffic sources, with heavy-tailed on or off periods, results in self-similar aggregated traffic [5]. The finding is important, as it indicates that heavy-tailed on-off periods can be the reason for self-similarity in TCP/IP 1 traffic. The result is confirmed in [6], which showed that individual data traffic source can be modelled by on-off sources with heavy-tailed on and off periods.

Unfortunately the network analysis with heavy-tailed traffic sources is very complicated. For example, in an M/G/1 queuing system, the average packet delay is proportional to the variance of the service time [7. In the presence of heavy-tailed on-off sources, the service time variance is infinite, which implies an infinite value for the average delay. Furthermore, it can be shown that, for on-off sources with heavy-tail on periods, the moment generation function (MGF) is infinite [7. This means that the Chernoff bound can not be used to analyze the queue delay performance. Jenkovic in 8 derived a relation among queue length distribution, transmission rate, and traffic characteristics for a single server system with a single on-off source in a wireline network. The analysis is carried out under the assumption that the network transmission rate (server capacity) is a constant. The assumption is not valid in wireless communications. Due to the time-variant fading dispersive propagation medium, the link capacity in a wireless system changes with time, and the transmission rate is not constant.

In this research, we study the queuing behavior of self-similar packet data traffic in a wireless single-server network, and derive a new relationship among the asymptotic distribution of queue length, traffic specifications, channel characteristics, and transmission rate. This study provides insights of the impact of the self-similar property on network resource allocation for QoS provisioning. The remainder of this paper is organized as follows. Section 2 presents mathematical definitions and describes the system model. Details of the queue analysis with self-similar input traffic is given in Section 3. Computer simulation results are presented in Section 4 to demonstrate the accuracy of the theoretical analysis. Conclusions are given in Section 5 .

\section{System Model}

We first present some mathematical definitions and concepts, which are used in this work.

Definition 1. 8. A cumulative distribution function (CDF) $F$ on $[0, \infty]$ is called heavy-tailed $(F \in \mathrm{E})$ if

$$
\lim _{x \rightarrow \infty} \frac{1-F(x-y)}{1-F(x)}=1, \quad y \in \Re .
$$

\footnotetext{
${ }^{1}$ Transmission Control Protocol/Internet Protocol.
} 
Definition 2. [8] A CDF function $F$ on $[0, \infty]$ is called subexponential $(F \in S)$ if

$$
\lim _{x \rightarrow \infty} \frac{1-F^{* 2}(x)}{1-F(x)}=2, \quad y \in \Re
$$

where $F^{* 2}(x)$ denotes the 2 nd convolution of $F$ with itself, i.e, $F^{* 2}(x)=\int_{0}^{+\infty} F(x-$ $y) F(y) d y$.

A well known example of subexponentially distributed functions is functions of regular variation $R_{\alpha}$ (in particular Pareto family); $F \in R_{\alpha}$ if it is given by

$$
F(x)=1-\frac{l(x)}{x^{\alpha}}, \quad \alpha \geq 0
$$

where $l(x): \Re_{+} \longrightarrow \Re_{+}$is a function of slow variation, i.e., $\lim _{x \rightarrow \infty} \frac{l(\delta x)}{l(x)}=1$.

In this work data traffic flows are modelled by on-off sources. An on-off source can be considered as a renewal process, with renewal periods of $\left(\tau_{n}^{\text {on }}+\tau_{n}^{\text {off }}, n \geq\right.$ 0 ), where $\tau_{n}^{\text {on }}$ and $\tau_{n}^{\text {off }}$ are the durations of on and off periods, respectively. The on periods follow a Pareto distribution and the off periods follow an exponential distribution. During each on period, packets are generated at a constant rate of $r$. The steady state probabilities of being in the on and off states are given by

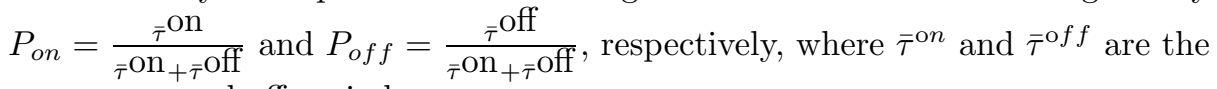
average on and off periods.

Consider transmission of data packets of equal length over a wireless channel. With a constant transmission information rate, the transmission times for all the packets are the same, referred to as packet time. An important QoS parameter in data service is the transmission accuracy. Consider the transmission over a wireless channel. The transmission accuracy can be described by the transmission bit error rate (BER). Given the modulation and coding scheme, the channel model and parameters, and the receiver structure, the required BER can be mapped one-to-one to the required received signal to interference-plus-noise ratio (SINR) 9]. For a given maximum transmit power, the required SINR can not be achieved and the data transmission stops if the channel is in a poor (deep fading) condition. When the channel condition improves over time (to a non deep fading condition), the required SINR can be ensured and the transmission over the channel can resume. As a result, we use a two-state channel model. The channel is said to be in a nonworking state in the former case and in a working state in the latter case. If the channel fades slowly with respect to the packet time, the channel state remains the same over a packet time. Given the channel fading statistics, the working-state probability $P_{w}$ and nonworking-state probability $P_{n w}$ can be calculated. The transmission system for each packet traffic flow can be described by a simple queueing model, where the input is a single on-off source and the packets are transmitted through a two-state fading channel, as illustrated in Figure 1, In the working state of the channel, the server transmits packets with a constant transmission rate of $C$, and in the nonworking state the server stops the transmission to save the resources. The scheduling principle based on the channel condition achieves high utilization efficiency of the limited 
radio resources and has been used in $3 \mathrm{G}$ wireless system design. As data traffic is in general not strictly delay sensitive, the QoS requirement for transmission delay can be specified by two parameters, $q_{t h}$ and $\epsilon$ : the probability that the queue length $Q$ at the scheduler exceeds the threshold $q_{t h}$ is less than a small value $\epsilon$, i.e., $P\left[Q>q_{t h}\right]<\epsilon$. Let $c(t)$ denote the time varying channel capacity to guarantee the required BER and transmission delay. Then, $c(t)=C$ if the channel is in a working state and $c(t)=0$ if the channel is in a nonworking state, at time $t$. The scheduler calculates and allocates the bandwidth of $c(t)$ by considering the channel status and traffic specifications, which are assumed to be known to the scheduler.

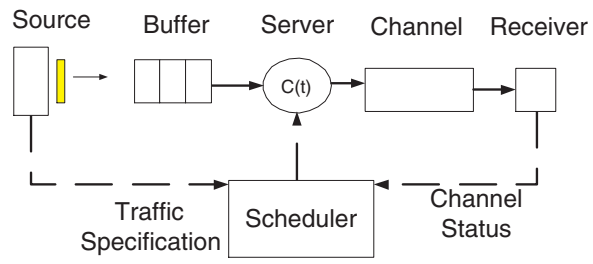

Fig. 1. Single server single input system

\section{Queuing Analysis}

We start the analysis by using a classical result given in 10. Let $a_{n}$ and $s_{n}$, $n \geq 0$, be two sequences of i.i.d. random variables, representing the number of packet arrival and departures in $n_{t h}$ recursion (with $\tau_{n}^{\text {on }}$ on period and $\tau_{n}^{\text {off }}$ off period of the source), respectively. Let $X_{n}\left(=a_{n}-s_{n}\right)$ denote the net increment of the packet number. In each recursion, the queueing process can be expressed as (Lindley recursion)

$$
Q_{n+1}=\left(Q_{n}+X_{n}\right)^{+}, \quad n \geq 0
$$

where $q^{+}=\max (q, 0)$, and $Q_{n}$ is the queue size at the beginning of the $n^{\text {th }}$ recursion. According to [10, this recursion admits a unique stationary solution and, for all initial conditions, the probability $P\left[Q_{n}>x\right]$ converges to the stationary probability of $P[Q>x]$ under stability condition $\bar{X}_{n}<0$ (where $\bar{X}_{n}$ denotes the expected value of $X_{n}$ ). In the following derivations, we assume that all the queuing systems under consideration are in their stationary regimes.

The residual life of a renewal process is defined as the duration between some fixed time $t$ and the starting point of the following renewal. It is one of the random variables that describe the local behavior of a renewal process. Another variable is the age at time $t$ [1], or the time already elapsed in the current renewal. When we look at a renewal process in the reverse (backward) direction, we again observe a renewal process having the same probability structure, but the residual life time is called age [11. Letting $F(x)$ denote the CDF of $X_{n}$, the $\mathrm{CDF}$ of the residual life, $F 1(x)$, is given by 


$$
F 1(x)=\frac{\int_{0}^{x}(1-F(u)) d u}{\bar{X}_{n}} .
$$

$F 1(x)$ is also called the integral tail distribution of $F(x)$.

Theorem 1 [8] If $F_{X}(x)$ is heavy-tailed and $F 1_{X}(x)$ is subexponential and $\bar{X}_{n}<0$, for (41) it can be shown that

$$
P\left[Q_{n}>x\right] \sim \frac{\int_{x}^{\infty} P\left[X_{n}>u\right] d u}{-\bar{X}_{n}}, \quad x \rightarrow \infty
$$

where $l(x) \sim j(x)$ denotes $l(x) / j(x)=1$ as $x \rightarrow \infty$.

Lemma 1 [8] Let $X$ and $Y$ be two independent random variables distributed as $F(x)$ and $G(x)$, respectively. If $F(x)$ is heavy tailed, $\bar{X}$ and $\bar{Y}$ are finite, and $Y$ is a positive non-heavy-tailed random variable, then

$$
P[X-Y>x] \sim P[X>x]=1-F(x), \quad x \rightarrow \infty .
$$

Consider the system illustrated in Figure 1, during the interval of $\Gamma_{n}=\tau_{n}^{\text {on }}+$ $\tau_{n}^{\mathrm{off}}$, the state of the channel may change several times, so does the capacity. We define $\Omega_{n}$ (in second) as the summation of all the time intervals (during the $\Gamma_{n}$ period) when the channel is in a working state. In a working state with capacity $C$, if there are backlogged packets in the queue, the number of the transmitted packets is $C \Omega_{n}$. We define the effective capacity of the time varying channel as $c_{n}=\frac{C \Omega_{n}}{\Gamma_{n}}$. Instead of considering the time-varying capacity, the effective capacity that is constant (even though random) during $\Gamma_{n}$ will be considered. As the PDF of $c_{n}$, denoted by $f_{c_{n}}(x)$, is unknown, we need to estimate the value of $c_{n}$ to proceed further.

Consider the evolution of the queue length at the initial moment of the on period, denoted by $Q_{n}^{p}$, where the superscript $p$ stands for Palm probability (meaning that the queue length is observed at the beginning of the on period). According to the definition of $X_{n}$, it can be shown that $X_{n}=r \tau_{n}^{\text {on }}-C \Omega_{n}$, and we have

$$
\begin{aligned}
Q_{n+1}^{p} & =\left(Q_{n}^{p}+X_{n}\right)^{+} \\
& =\left(Q_{n}^{p}+r \tau_{n}^{\text {on }}-C \Omega_{n}\right)^{+} \\
& =\left(Q_{n}^{p}+r \tau_{n}^{\text {on }}-c_{n}\left(\frac{\Gamma_{n}}{\Omega_{n}}\right) \Omega_{n}\right)^{+} \\
& =\left(Q_{n}^{p}+r\left(\tau_{n}^{\text {on }}\right)-c_{n}\left(\tau_{n}^{\text {on }}+\tau_{n}^{\text {off }}\right)\right)^{+} \\
& =\left(Q_{n}^{p}+\tau_{n}^{\text {on }}\left(r-c_{n}\right)-c_{n} \tau_{n}^{\text {off }}\right)^{+}
\end{aligned}
$$

where $r-c_{n}>0$.

With a Pareto distributed on period and an exponentially distributed off period, we have

$$
P\left[\tau_{n}^{\text {on }}>x\right]=\frac{b}{x^{\alpha}}, \quad x \geq 0
$$

and

$$
P\left[\tau_{n}^{\text {off }}>x\right]=e^{-\lambda x}, \quad x \geq 0 .
$$


By Lemma 1, for $x \rightarrow \infty$, we have

$$
\begin{aligned}
P\left[X_{n}>x\right] & =P\left[\tau_{n}^{\text {on }}\left(r-c_{n}\right)-c_{n} \tau_{n}^{\text {off }}>x\right] \\
& \sim P\left[\tau_{n}^{\text {on }}\left(r-c_{n}\right)>x\right] .
\end{aligned}
$$

Since

$$
P\left[X_{n}>x\right]=E_{c_{n}}\left(P\left[X_{n}>x \mid c_{n}\right]\right)
$$

we have

$$
\begin{aligned}
P\left[X_{n}>x\right] & \sim E_{c_{n}}\left(P\left[\tau_{n}^{\text {on }}\left(r-c_{n}\right)>x \mid c_{n}\right]\right) \\
& =E_{c_{n}}\left[\frac{b\left(r-c_{n}\right)^{\alpha}}{x^{\alpha}}\right] \\
& =\frac{b k}{x^{\alpha}}
\end{aligned}
$$

where $k=E_{c_{n}}\left[\left(r-c_{n}\right)^{\alpha}\right]$. Equation (10) shows that $X_{n}$ also has a Pareto distribution. Under the assumption that the on and off periods are stationary, it can be shown that

$$
\begin{aligned}
\bar{X}_{n} & =E_{c_{n}}\left(\bar{\tau}_{n}^{\text {on }}\left(r-c_{n}\right)-c_{n} \bar{\tau}_{n}^{\text {off }}\right) \\
& =r \bar{\tau}^{\text {on }}-\left(\bar{\tau}^{\text {on }}+\bar{\tau}^{\text {off }}\right) \bar{c}_{n} .
\end{aligned}
$$

Assuming that $c_{n}$ is an ergodic process, we have $\bar{c}_{n}=C P_{w}$ and

$$
\bar{X}_{n}=r \bar{\tau}^{\text {on }}-\left(\bar{\tau}^{\text {on }}+\bar{\tau}^{\text {off }}\right) C P_{w} .
$$

Considering the stability condition $\bar{X}_{n}<0$, it is required that

$$
\frac{r \bar{\tau} \text { on }}{\bar{\tau}^{\text {on }}+\bar{\tau}^{\text {off }}}<C P_{w} \text {. }
$$

As a large queue length is mainly the result of large $\Gamma_{n}$ values (large bursts), the probability of $P[Q>x]$ for a large $x$ depends mainly on large $\Gamma_{n}$ values. When $\Gamma_{n}$ is large, we can assume that $\frac{\Omega_{n}}{\Gamma_{n}} \simeq P_{w}$ and the effective capacity $c_{n} \simeq C P_{w}$. In the case of a large queue, $X_{n}$ can be approximately estimated by

$$
X_{n} \simeq \tau_{n}^{\text {on }}\left(r-C P_{w}\right)-C P_{w} \tau_{n}^{\text {off }}
$$

for large $X_{n}$ values. Due to the fact that the distribution of $X_{n}$ is heavy tailed, by Theorem 1 , it can be shown that

$$
P\left[Q^{p}>x\right] \sim \frac{b k}{\left[C P_{w}\left(\bar{\tau}^{\text {on }}+\bar{\tau}^{\text {off }}\right)-r \bar{\tau}^{\text {on }}\right](\alpha-1) x^{\alpha-1}}, \quad x \rightarrow \infty .
$$

Substituting $k=E_{c_{n}}\left(r-c_{n}\right)^{\alpha} \simeq\left(r-C P_{w}\right)^{\alpha}$,

$$
P\left[Q^{p}>x\right] \approx \frac{b\left(r-C P_{w}\right)^{\alpha}}{\left[C P_{w}\left(\bar{\tau}^{\mathrm{o} n}+\bar{\tau}^{\mathrm{off}}\right)-r \bar{\tau}^{\mathrm{o} n}\right](\alpha-1) x^{\alpha-1}}, \quad x \rightarrow \infty
$$


where $l(x) \approx j(x)$ denotes $l(x) / j(x) \simeq 1$ as $x \rightarrow \infty$. The stationary probability $P[Q>x]$ can be calculated from the Palm queue in (16).

Let $\Lambda_{n}^{\text {on }},-\infty<n<\infty$, be a random process that represents the beginning of the on periods in the stationary on-off process with $\Lambda_{0}^{\text {on }}<0 \leq \Lambda_{1}^{\text {on }}$, and $B$

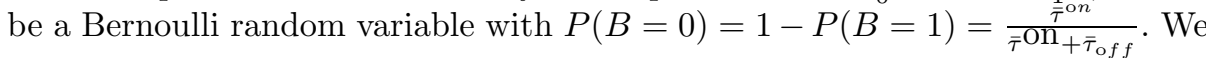
define the residual on period, $\tau_{n}^{\text {on, } r}$, as the residual life time of $\tau_{n}^{\text {on }}$ with respect to time 0 . Similarly, the residual off period, $\tau_{n}^{o f f, r}$, can be defined. $\Lambda_{0}^{\text {on }}$ can be represented by

$$
-\Lambda_{0}^{\mathrm{on}}=B\left(\tau_{0}^{\mathrm{on}}+\tau_{0}^{\mathrm{off}, \mathrm{r}}\right)+(1-B) \tau_{0}^{\mathrm{on}, \mathrm{r}} .
$$

The preceding equation means that, if the source is in the on state $(B=0)$ at time $t=0,-\Lambda_{0}^{\mathrm{On}}$ is the age (residual life) of the on period, $\tau_{0}^{\mathrm{on}, \mathrm{r}}$; otherwise, $-\Lambda_{0}^{\mathrm{On}}$ is the duration of the on period plus the age (residual life) of the off period. Furthermore, the net increment $X_{0}$ of the load that arrives to the queue in the interval $\left[\Lambda_{0}^{\mathrm{on}}, 0\right]$ is equal to

$$
X_{0}=B\left[\tau_{0}^{\text {on }}\left(r-c_{0}\right)-c_{0} \tau_{0}^{\text {off, }, \mathrm{r}}\right]+(1-B)\left[\left(r-c_{0}\right) \tau_{0}^{\text {on }, \mathrm{r}}\right]
$$

Let $Q_{T_{0}}$ denote the backlogged traffic observed at the beginning of the zero ${ }^{\text {th }}$ renewal period $\left(t=\Lambda_{0}^{\text {on }}\right)$. The asymptotic probability of the queue length in the zero $^{\text {th }}$ renewal period, $P\left[Q_{0}>x\right]$, is

$$
\begin{array}{rl} 
& P\left[Q_{0}>x\right] \\
=P & P\left[Q_{0}>x, B=1\right] P[B=1]+P\left[Q_{0}>x, B=0\right] P[B=0] \\
= & P\left[Q_{T_{0}}+\left(r-c_{0}\right) \tau_{0}^{\text {on }}-c_{0} \tau_{0}^{\text {off, }}>x\right] P[B=1] \\
& \quad+P\left[Q_{T_{0}}+\left(r-c_{0}\right) \tau_{0}^{\text {on }}, \mathrm{r}>x\right] P[B=0] .
\end{array}
$$

Note that $Q_{T_{0}}$ is equal to the Palm queue $Q_{0}^{p}$. Since $Q_{T_{0}}$ and $\tau_{0}^{\text {on,r }}$ are subexponentially distributed, it can be shown that 8

$$
\left[Q_{T_{0}}+\left(r-c_{0}\right) \tau_{0}^{\mathrm{on}, \mathrm{r}}>x\right] \sim P\left[Q_{T_{0}}>x\right]+P\left[\left(r-c_{0}\right) \tau_{0}^{\mathrm{on}, \mathrm{r}}>x\right], x \rightarrow \infty
$$

Also by Lemma 1 ,

$$
P\left[Q_{T_{0}}+\left(r-c_{0}\right) \tau_{0}^{\text {on }}-c_{0} \tau_{0}^{\text {off, }}>x\right] \sim P\left[Q_{T_{0}}>x\right], \quad x \rightarrow \infty .
$$

Equation (19) can then be simplified by using (20) and (21),

$$
P\left[Q_{0}>x\right] \sim P\left[Q_{T_{0}}>x\right]+P\left[\left(r-c_{0}\right) \tau_{0}^{\text {on, } \mathrm{r}}>x\right] P(B=0), x \rightarrow \infty .
$$

Considering that $P\left[\tau_{0}^{\text {on }}>x\right]=\frac{b}{x^{\alpha}}$ for $x \geq 0$, we have, for $x \geq 0$,

$$
P\left[\left(r-c_{0}\right) \tau_{0}^{\text {on }, \mathrm{r}}>x\right]=E_{c_{0}}\left(\frac{\int_{\frac{x}{r-c_{0}}}^{\infty} \frac{b}{u^{\alpha}} d u}{\bar{\tau}_{0}^{\mathrm{On}}}\right)=E_{c_{0}}\left(\frac{b\left(r-c_{0}\right)^{\alpha-1}}{\bar{\tau}_{0}^{\mathrm{on}}(\alpha-1) x^{\alpha-1}}\right) .
$$


Using $c_{0} \simeq C P_{w}$, we have

$$
P\left[\left(r-c_{0}\right) \tau_{0}^{\mathrm{on}, \mathrm{r}}>x\right] \simeq \frac{b\left(r-C P_{w}\right)^{\alpha-1}}{\bar{\tau}_{0}^{\mathrm{on}}(\alpha-1) x^{\alpha-1}} .
$$

With $Q_{T_{0}}=Q_{0}^{p}$ and $Q$ being stationary (i.e., $P\left[Q_{0}>x\right]=P\left[Q_{n}>x\right]=P[Q>$ $x]$ ), applying (15) and (24) to (22), we have

$$
\begin{gathered}
P[Q>x] \approx P\left[Q_{0}^{p}>x\right]+\left(\frac{1}{\left.\bar{\tau}^{\text {on }}+\bar{\tau}^{\text {off }}\right)}\right) \frac{b\left(r-C P_{w}\right)^{\alpha-1}}{(\alpha-1) x^{\alpha-1}} \\
\approx \frac{b\left(r-C P_{w}\right)^{\alpha}}{\left(C P_{w}\left(\bar{\tau}^{\text {on }}+\bar{\tau}^{\text {off }}\right)-r \bar{\tau}^{\text {on }}\right)(\alpha-1) x^{\alpha-1}} \\
\quad+\frac{b\left(r-C P_{w}\right)^{\alpha-1}}{\left(\bar{\tau}^{\text {on }}+\bar{\tau}^{\text {off }}\right)(\alpha-1) x^{\alpha-1}}, \quad x \rightarrow \infty .
\end{gathered}
$$

In (25), we estimate the asymptotic behavior of $\mathrm{Q}$ for a large $x$. By using this relationship, we are able to calculate the required link capacity (transmission rate) $C$ based on traffic parameters $\left(r, \bar{\tau}_{n}^{\text {on }}, \bar{\tau}_{n}^{\text {off }}, b, \alpha\right)$ and channel characteristics $\left(P_{w}\right)$ to reach a predefined QoS level (in terms of delay specified by $q_{t h}$ and $\epsilon$ ).

\section{Simulation}

Due to the complexity of the queue analysis with the self-similar input traffic flow, we have to make several simplified assumptions for tractability in deriving (25). To validate the analysis given in Section 3, computer simulations were carried out and the results are presented in the following.

The single server single input system illustrated in Figure1 is simulated. The information bits of the input traffic flow are generated during each on period at a rate of $r$. The on periods are determined from the sample values of the Pareto random variable, generated by using the random generator as specified in [12. The off periods are determined from the sample values of the exponential random variable. As the data packets have the same length and are transmitted at a constant rate, without loss of generality, we simulate the generation and transmission of bit flows instead of packet flows. The simulation parameters are chosen based on the previous measurements for FTP application in wireless networks [13] to be $\bar{\tau}^{\text {on }}=0.256 \mathrm{~s}, \bar{\tau}^{\text {off }}=15 \mathrm{~s}, r=64 \mathrm{kbps}$, and frame duration of $10 \mathrm{~ms}$. Although $\alpha=1.1$ is suggested in [13, we choose $\alpha=1.4$ in the simulation, because the accuracy of the Pareto random generator is relatively poor for a small $\alpha$ value. The two-state channel status is characterized by a Bernoulli random process with working probability $\left(P_{w}\right)$, changes independently from frame to frame. Each run in the simulations consists of at least $10 \mathrm{e} 8$ frames.

Figure 2 shows the tail distribution of the queue length for the buffer, with $P_{w}$ being 0.5 and 0.9 respectively and capacity $C$ of $40 \mathrm{kbps}$. The analytical results are obtained from (25). It is observed that the simulation results agree very well with the analytical results. 


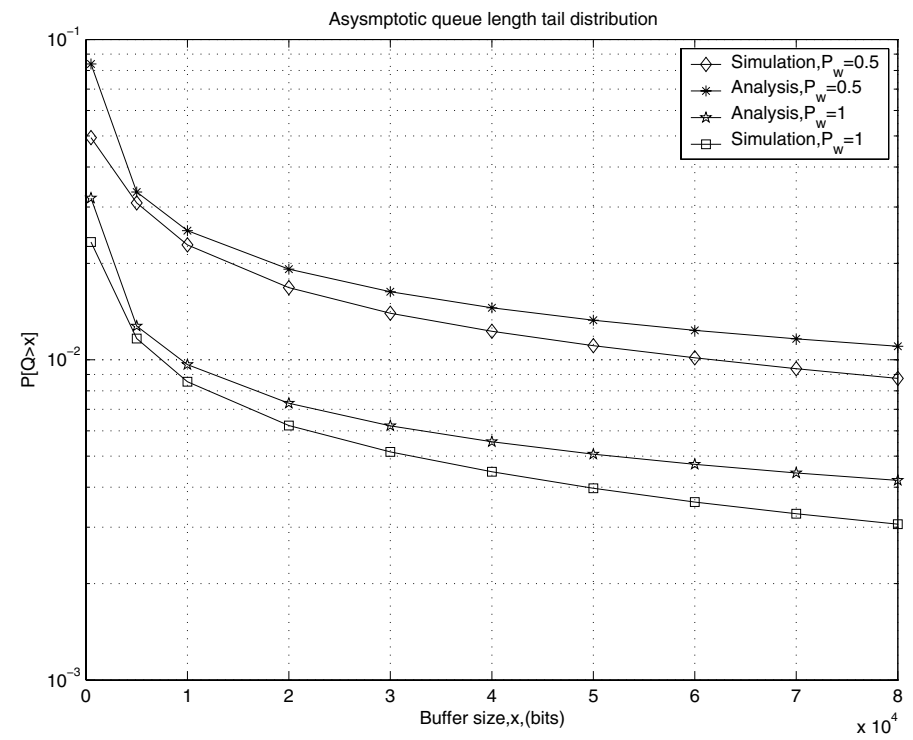

Fig. 2. Effect of the wireless channel quality on the queue length distribution

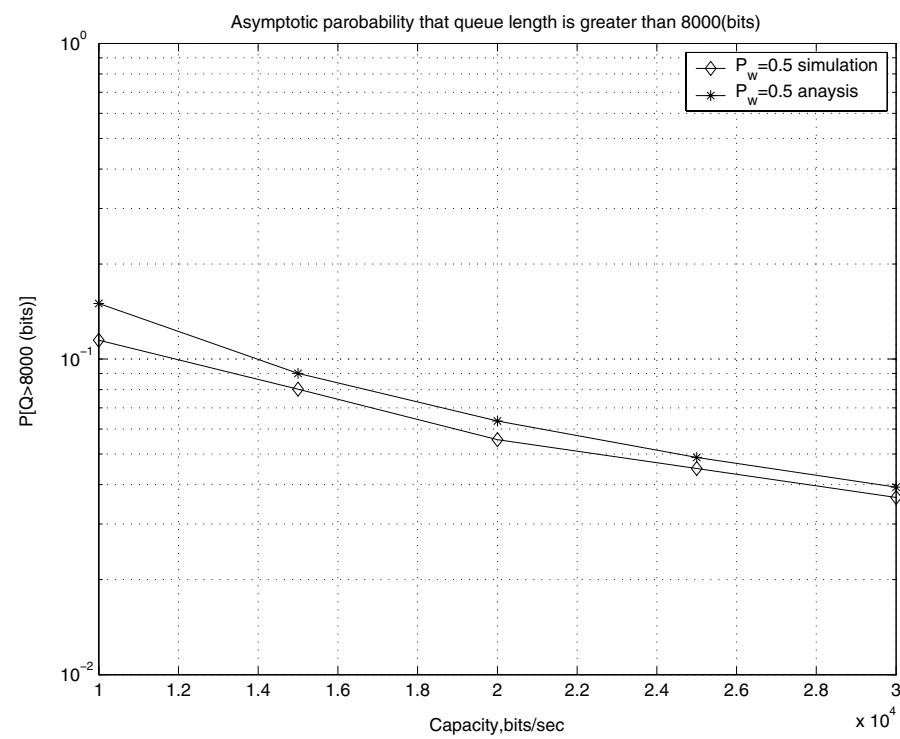

Fig. 3. Effect of the capacity on the queue length distribution

Figure 3 plots the probability, $P[Q>8000$ (bits)], as a function of the system capacity $C$ with $P_{w}$ being 0.5 . Again, we observe a close agreement between the analytical results obtained from (25) and the simulation results. 


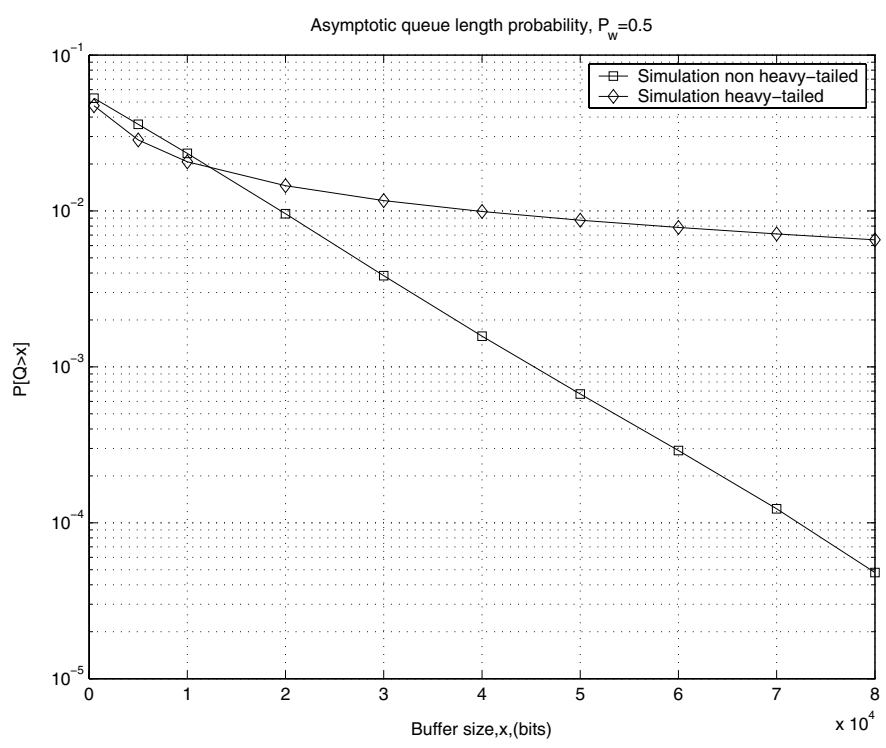

Fig. 4. Impact of the traffic model on the queue length distribution

Figure 4 shows the impact of the heavy-tailed traffic on the asymptotic taildistribution of queue length. Two traffic flows, with the same average rate and equal expected on and off periods, are simulated respectively. One is a non heavytailed on-off source, where both on and off periods are exponentially distributed. The other is a heavy-tailed traffic, whose on periods have a Pareto distribution with $\alpha=1.4$. The system parameters are the same for both inputs with $C=40$ kbps and $P_{w}=0.5$. The distribution for the non heavy-tailed traffic declines very fast (exponentially), but not in the case of the heavy-tailed source. It is the main and the most important impact of the heavy-tailed traffic flows on network performance. It is observed that the distribution for the heavy-tailed traffic has a relatively large value even for a very large queue length, which causes the possibility of a very large delay and a large probability of packet loss.

\section{Conclusions}

This paper investigates the impact of self-similarity property of the source traffic flow on queue length distribution in a wireless system having a single input and a single server. The wireless channel is characterized by an i.i.d two-state model. The close-form expression is derived for the relation among the traffic parameters, the channel working state probability, the server capacity, and the queue length distribution. The derivation extends the research presented in [8] for wireline networks to a wireless communication environment. The preliminary simulation results demonstrate that the assumptions made in the analysis are reasonable and the derived relation given in (25) is accurate. 


\section{Acknowledgement}

This research was supported by Bell University Labs (BUL) and Communications Information Technology Ontario (CITO).

\section{References}

1. V. Paxson and S. Floyd, "Wide area traffic: the failure of poisson modeling," IEEE/ACM Transactions on Networking, vol. 3, pp. 226-244, June 1995.

2. M. Crovella and A. Bestavros, "Self-similarity in world wide web traffic: evidence and possible causes," IEEE/ACM Transactions on Networking, vol. 5, pp. 835-846, December 1997.

3. M. Jiang, M. Nikolic, S. Hardy, and L. Trajkovic, "Impact of self-similarity on wireless data network performance," in Proc. IEEE ICC'01, vol. 2, pp. $477-481$, June 2001.

4. M. Cheng and L.F. Chang, "Wireless dynamic channel assignment performance under packet data traffic", IEEE J. Select. Areas Commun., vol. 17, no. 7, pp. 1257-1269, July 1999.

5. W. Willinger, M. Taqqu, R. Sherman, and D. V. Wilson, "Self-similarity through high-variability: statistical analysis of Ethernet LAN traffic at source level at source level," IEEE/ACM Trans. Networking, vol. 5, pp. 71-86, February 1997.

6. K. Park, G. Kimy, and M. Crovellaz, "On the relationship between file sizes, transport protocols, and self-similar network traffic," Proc. of the Fourth International Conference on Network Protocols (ICNP'96), October 1996.

7. P. Jelenkovic, "Heavy tails and fluid queues: lecture notes." http://comet.ctr.columbia.edu/ anar/e6762/e6762.htm, 2002.

8. P.R. Jelenkovic and A.A. Lazar, "Multiplexing on-off sources with sub exponential on periods: part1," in Proc. IEEE/ACM Infocom'97, 1997.

9. B. Collins and R. Cruz, "Transmission policies for time varying channels with average delay constraints," in Proc. 1999 Alleron Conf. Communication, Control, E Computer, 1999.

10. R.M. Loynes, "The stability of a queue with non independent inter arrival and service time," in in Proc. Cambridge Philosophy Society, vol. 58, pp. 496-520, 1968.

11. S.M. Ross, Introduction to Probability Models, 8th ed. Academic Press, 2003.

12. G. Kramer, "Self-similar network traffic," Dept. of Computer Science University of California, http://wwwcsif.cs.ucdavis.edu/ kramer/research.html, 2001.

13. G. Iacovoni, "Wg2 interaction," Tech. Rep., Ericsson Lab, Italy, 1998. 